Nachhaltigkeitsberichterstattung ist auf dem Weg

\section{Kreativität oder Norm?}

\author{
In der Entwicklung der Berichterstattung von Unternehmen zeichnet sich immer \\ deutlicher ein Trend zum integrierten Reporting über die drei Dimensionen der \\ Nachhaltigkeit ab. Dies gilt in Deutschland wie weltweit. Nachhaltigkeitsbe- \\ richterstattung ist jedoch als Entwicklungsprozess zu betrachten. Er kann einer- \\ seits durch eine zu frühe oder zu stringente Standardisierung gehemmt werden, \\ andererseits durch eine Vereinfachung mit Hilfe von Leitlinien für Unternehmen \\ erheblich erleichtert und so schneller vorangetrieben werden. Gesucht ist, wie \\ so häufig, der goldene Mittelweg.
}

I Von Michaela Raupach und Jens Clausen m Zusammenhang mit der zunehmenden Verbreitung des Nachhaltigkeitsgedankens in Gesellschaft und Unternehmen verändert sich auch die Art der Berichterstattung von Unternehmen. Besonders große, multinationale Unternehmen gehen immer häufiger dazu über, anstelle von getrennten Sozial- und Umweltberichten einen Nachhaltigkeitsbericht zu erstellen. Dieser stellt die verschiedenen Dimensionen unternehmerischen Handelns - also ökonomische, ökologische und soziale Aspekte der Unternehmenstätigkeit - integriert dar und hebt dabei auch die zwischen den Dimensionen bestehenden Wechselwirkungen, Synergien und Zielkonflikte hervor. Ein Nachhaltigkeitsbericht ist demnach mehr als lediglich eine Zusammenfassung oder die Summe der drei Einzelaspekte. Er zeichnet sich durch eine ganzheitliche, integrierte Darstellungsweise aus. Das Unternehmen kann Entwicklungsperspektiven herausarbeiten und Fortschritte auf seinem Weg zur Umsetzung der NachhaltigkeitsPrinzipien aufzeigen.

Eine große Zahl von Anspruchsgruppen ist von den Auswirkungen der Unternehmenstätigkeit und damit letztlich auch vom Bericht betroffen. Dieser sollte daher auch darstellen, wie das Unternehmen im Dialog zu diesen Gruppen steht, welche gemeinsamen Planungsprozesse betrieben werden und welche Widersprüche und Beschwerden es gibt. Ein Nachhaltigkeitsbericht kann so auch dem innerhalb des Unternehmens sowie zwischen Unternehmen und Anspruchsgruppen ablaufenden Integrationsprozess dienen.

Die gestiegenen Anforderungen der Gesellschaft an die Berichterstattung der Unternehmen sowie der zunehmende Bedarf an dreidimensionaler
Information über die Leistung und Auswirkungen des Unternehmens auf Umwelt und Gesellschaft führten 1998 zur Gründung der Global Reporting Initiative (GRI). Diese präsentierte im März 1999 den ersten öffentlichen Entwurf eines Leitfadens für Nachhaltigkeitsberichte, der derzeit bereits in einer überarbeiteten zweiten Version vorliegt (1). Ziel der Initiative ist es, aufbauend auf der Praxis der Umweltberichterstattung die sozialen und ökonomischen Aspekte zu integrieren, so dass schließlich über alle drei Dimensionen der Nachhaltigkeit und deren Interdependenzen integriert berichtet wird. 21 internationale Kon-

Neue Ansätze der
Ökonomie aus
(sozial-)ökologischer
Perspektive
und
Governance und
Nachbaltigkeit
mit Spezial: Wo bleibt
die Energiewende?
sind die Themen
der Informationsdienste
Okologisches Wirtschaften
2/2001 und 3-4/2001
Wenn Sie potenzielle Beiträge
haben, wenden Sie sich bitte
an die Redaktion.

zerne beteiligen sich an der Erprobung der Umsetzung von Leitlinien und vorgegebenen Indikatoren und Kennzahlen.

Zusätzlichen Rückenwind dürfte auch die Neufassung der OECD-Leitsätze für multinationale Unternehmen geben, in denen die Offenlegung ökologischer und sozialer Informationen empfohlen wird. Auch in der Europäischen Kommission wird über „Corporate Social Responsibility Reporting“ nachgedacht.

Trotz dieser global zu beobachtenden Entwicklungen besteht jedoch bei weitem noch kein Konsens darüber, welche Inhalte und Darstellungen die Bezeichnung eines Unternehmensberichtes als Nachhaltigkeitsbericht rechtfertigen. Auch bei der Umweltberichterstattung - die seit Beginn der neunziger Jahre zunehmend verbreitet ist - hat aber die Herausbildung einer weitgehenden Übereinstimmung zu diesen Fragen einige Jahre in Anspruch genommen.

\section{Der Stand der Dinge}

Zur Zeit sind also viele Faktoren, die für das Thema Nachhaltigkeitsberichterstattung von Unternehmen von Bedeutung sind, noch im Umbruch:

- Unklar ist häufig, über welche spezifischen Aspekte der jeweiligen Nachhaltigkeits-Dimensionen berichtet werden sollte. Beispielsweise ist im ökonomischen Bereich umstritten: soll hier der Nukleus einer verantwortlichen, langfristigen Ökonomie betont werden oder sind konventionelle Argumente gewinnorientierter Ökonomie die Brücke zur Nachhaltigkeit, über die auch klassische Manager gehen können?

- Große Kontroversen ruft insbesondere im sozialen Bereich die Bestimmung und Festlegung von Messgrößen und Kennzahlen hervor. Besonders bei Rating-Agenturen, aber auch bei anderen Akteuren besteht der Wunsch, branchenübergreifend und international anwendbare Kennzahlen zur Messung der Leistung eines Unternehmens zu haben. Dem stehen aber von Branche zu Branche völlig unterschiedliche relevante Themen, unterschiedliche Fertigungstiefen und von Land zu Land unterschiedliche Rahmenbedingungen entgegen (vgl. auch den Beitrag von Lohrie).

- Vollends problematisch: Wie kann die Integration der drei Dimensionen in die Unternehmensabläufe, die Berïcksichtigung der bestehenden Wechselwirkungen, Synergien und Zielkonflikte bei unternehmerischen Entscheidungen, 
also die tatsächliche Ausrichtung des Unternehmens am Konzept der Nachhaltigkeit gezeigt und gemessen werden? Hier sollten sich Leser und Unternehmen an eine Darstellung mit Hilfe von Texten, Argumentationen und Beispielen gewöhnen. Zwar gibt es erste Ansätze für sogenannte integrierte Kennzahlen - wie beispielsweise die Öko-Effizienz, welche die Dienstleistungseinheit pro Einheit Umwelteinwirkung darstellt und so die wirtschaftliche Dimension mit der ökologischen verknüpft. Das Sammeln von Erfahrungen mit solchen Indikatoren und Kennzahlen sowie deren Weiterentwicklung und Optimierung wird jedoch noch einige Zeit in Anspruch nehmen.

- Auch zur Frage der Verpflichtung von Unternehmen zur Berichterstattung gibt es unterschiedliche Auffassungen. Zumindest für die nächsten Jahre ist wohl auf Freiwilligkeit zu setzen, denn international ist kaum eine Berichterstattungspflicht durchsetzbar, und gerade seitens der internationalen Unternehmen ist die Rechenschaftslegung besonders drängend.

Diese Schwierigkeiten werden auch im kürzlich veröffentlichten, ersten internationalen Ranking von Nachhaltigkeitsberichten deutlich (2). Untersucht wurden von SustainAbility Ltd. und der UN-Umweltorganisation UNEP 200 Berichte, die größtenteils auf den Bereich Umwelt, zu kleineren Teilen auch auf die soziale Dimension oder explizit auf Nachhaltigkeit fokussieren. Dabei fanden sich nur wenige Berichte, die als Ansatz zum „Triple-Bottom-Line-Reporting“ bezeichnet werden können. Hierzu gehören die Unternehmen, die im Ranking die höchste Punktzahl erreichen: British Airport Administration (Flughäfen) und Novo Nordisk (Pharmazie/ Gentechnische Herstellung von Enzymen). Trotzdem fehlen auch in diesen Berichten entscheidende Elemente: Während Novo Nordisk auch umstrittene Themen zur Diskussion stellt, dafür aber die ökonomische Dimension nicht in die Nachhaltigkeits-Diskussion integriert, werden im Bericht der BAA die fundamentalsten Aspekte der Unternehmenstätigkeit, nämlich die Auswirkungen des zunehmenden Flugverkehrs auf Umwelt und Gesellschaft, nicht erkannt - oder zumindest nicht genannt.

\section{Wohin geht die Reise?}

Die (Weiter-)Entwicklung eines einheitlichen, nachvollziehbaren und umsetzbaren Systems für die Berichterstattung von Unternehmen über Nachhaltigkeit ist ein Prozess, der durch Erfahrungen sowohl von den Unternehmen selbst als auch von deren Stakeholdern begleitet und ergänzt werden muss. Eine zu frühe und zu stringente Standardisierung birgt nach Ansicht vieler Unternehmen und Forschungsinstitute die Gefahr, dass wichtige Entwicklungsschritte ausbleiben und die Motivation der Unternehmen zur Berichterstattung über Nachhaltigkeit gemindert wird. Die bisher noch bestehenden Freiheiten der Unternehmen in Bezug auf Umfang, Inhalt und Gestaltung des Nachhaltigkeitsberichts könnten sich zwar einerseits nachteilig auf die Vergleichbarkeit der Berichte untereinander auswirken sowie der Aufmachung der Berichte als Image- oder Werbebroschüre Vorschub leisten.

Andererseits muss auch das Ziel im Blickfeld bleiben, möglichst viele Unternehmen zur Erstellung eines solchen Berichts zu motivieren und damit wiederum die Weiterentwicklung der Nachhaltigkeitsberichterstattung zu fördern. Dies kann jedoch nur gelingen, wenn die Unternehmen sich von der Veröffentlichung eines Nachhaltigkeitsberichts Image- bzw. Wettbewerbsvorteile versprechen. Hierbei ist auch die Unternehmensgröße von Bedeutung: Während in Großunternehmen ein Umwelt- oder Nachhaltigkeitsbericht als spezielles Kommunikationsinstrument betrachtet wird, nutzen kleinere Unternehmen Umwelt- und Nachhaltigkeitsberichterstattung oft auch zur Selbstdarstellung. Wenn also kleine oder mittelständische Unternehmen Nachhaltigkeitsberichte als ,informationshaltige Imagebroschüren“ gestalten, so entspringt dies dem Zwang, knappe Kommunikationsmittel effizient zu nutzen. Zwar wird so die reine Rechenschaftslegung verwässert, dafür steigt aber die Chance, auch kleinere Unternehmen für die Idee zu gewinnen und so die Verbreitung der Idee der Nachhaltigkeit zu unterstützen.

Sowohl für als auch gegen die Standardisierung der Nachhaltigkeitsberichterstattung sprechen schlagkräftige Argumente. Einerseits steht die plausible Forderung nach vergleichbaren, aussagekräftigen Berichten im Raum. Zum jetzigen Zeitpunkt ist jedoch ein allgemein anerkannter, einheitlich anzuwendender Standard einer Weiterentwicklung des Themas womöglich wenig förderlich: $\mathrm{Zu}$ strittig sind noch die innerhalb der einzelnen Dimensionen abzudeckenden Themenfelder, die Leistungsmessung und vor allem die Schwierigkeit der Darstellung Dimensionen übergreifender Aspekte. Unsicherheit herrscht auch darüber, wie eine solche Themenvielfalt den Lesern ansprechend und verständlich präsentiert werden kann.

\section{- Konkrete Schritte gefragt}

Praktikabel und hilfreich könnte jedoch zumindest die Bereitstellung von konkreten Handlungs- und Anwendungsvorschlägen zur Erstellung eines Nachhaltigkeitsberichts sein. Dies gilt insbesondere für kleinere und mittelständische Unternehmen, die bisher mit dieser Form der Berichterstattung eher wenig Erfahrung haben. Als Ergebnis des Projekts ,Schritte zur Nachhaltigkeitsberichterstattung", welches derzeit gemeinsam vom Institut für ökologische Wirtschaftsforschung (IÖW) und Institut für Markt Umwelt Gesellschaft (imug) in Kooperation mit vier Unternehmen durchgeführt wird (3), soll im Frühjahr ein Leitfaden vorliegen. In ihm werden auf Basis vieler Praxisbeispiele Anregungen gegeben, wie unter Maximierung des Nutzens für Anspruchsgruppen und Unternehmen die schwierige Aufgabe der Erstellung und Gestaltung eines Nachhaltigkeitsberichts gelöst werden kann. Dabei sollte der Leitfaden eher als Anstoß zur Kreativität denn als Ansatz der Vereinheitlichung verstanden werden.

Gleichzeitig wird rund um die GRI auch die Debatte um Kennzahlen, Gliederungen und Grundsätze von Nachhaltigkeitsberichten fortgefuihrt (4).

\section{Anmerkungen}

(1) Vgl. Global Reporting Initiative: Sustainability Reporting Guidelines. Boston 2000. Siehe hierzu auch Ökologisches Wirtschaften 5/00, S. 2 und 4.

(2) SustainAbility Ltd.: The Global Reporters. London 2000. Siehe hierzu auch die Meldung auf S. 3 dieses Heftes.

(3) Vgl. Clausen, Jens/ Häßler, Rolf/ Klaffke, Kathrin/Loew, Thomas/Raupach, Michaela/Schoenheit, Ingo unter Mitarbeit von Klaus Fichter: Schritte zur Nachhaltigkeitsberichterstattung Zwischenbericht zum internationalen Stand, Erfahrungen aus der Umwelt- und Sozialberichterstattung und zum Informationsbedarf der Zielgruppen. Diskussionspapier des lÖW 50/00, Berlin 2000. (4) Ihr kann im Internet unter http://www.globalreporting.org gefolgt werden. Dort findet sich als Debattenbeitrag auch imug/ IÖW/ifeu/Öko-Institut: Positionspapier deutscher Umwelt-Institute zum GRI-Leitfaden für Nachhaltigkeitsberichte.

\section{Die Autorlnnen}

Michaela Raupach ist wissenschaffliche Mitarbeiterin am Institut für Markt-Umwelt-Gesellschaft e.V. Kontakt: imug, Escherstr. 23, 30159 Hannover, Tel.: 0511/ 91115-0, Fax -95, E-mail: raupach@ imug.de

Jens Clausen ist wissenschaftlicher Mitarbeiter am Institut für ökologische Wirtschaftsforschung Kontakt: IÖW-Projektbüro Hannover, Hausmannstr. 9-10, 30159 Hannover. Tel. 0511/ 16403-44, Fax -91, E-mail: Jens.Clausen@hannover.ioew.de 
(c) 20I0 Authors; licensee IÖW and oekom verlag. This is an article distributed under the terms of the Creative Commons Attribution Non-Commercial No Derivates License (http://creativecommons.org/licenses/by-nc-nd/3.o/), which permits unrestricted use, distribution, and reproduction in any medium, provided the original work is properly cited. 